\title{
WEAK AND VISCOSITY SOLUTIONS OF THE FRACTIONAL LAPLACE EQUATION
}

\author{
RAFFAella SERVAdei AND EnRICO VAldinoci
}

\begin{abstract}
Aim of this paper is to show that weak solutions of the following fractional Laplacian equation

$$
\begin{cases}(-\Delta)^{s} u=f & \text { in } \Omega \\ u=g & \text { in } \mathbb{R}^{n} \backslash \Omega\end{cases}
$$

are also continuous solutions (up to the boundary) of this problem in the viscosity sense.

Here $s \in(0,1)$ is a fixed parameter, $\Omega$ is a bounded, open subset of $\mathbb{R}^{n}(n \geqslant 1)$ with $C^{2}$-boundary, and $(-\Delta)^{s}$ is the fractional Laplacian operator, that may be defined as

$$
(-\Delta)^{s} u(x):=c(n, s) \int_{\mathbb{R}^{n}} \frac{2 u(x)-u(x+y)-u(x-y)}{|y|^{n+2 s}} d y,
$$

for a suitable positive normalizing constant $c(n, s)$, depending only on $n$ and $s$.

In order to get our regularity result we first prove a maximum principle and then, using it, an interior and boundary regularity result for weak solutions of the problem.

As a consequence of our regularity result, along the paper we also deduce that the first eigenfunction of $(-\Delta)^{s}$ is strictly positive in $\Omega$.
\end{abstract}

2010 Mathematics Subject Classification: 35R09, 45K05, 49N60, 35D30.

Key words: Integrodifferential operators, fractional Laplacian, weak solutions, viscosity solutions, regularity theory.

We are indebted to Ovidiu SAvin, JoAquim Serra and Luis Silvestre for several pleasant and fruitful discussions.

The first author was supported by the MIUR National Research Project Variational and Topological Methods in the Study of Nonlinear Phenomena and the GNAMPA Project Variational Methods for the Study of Nonlocal Elliptic Equations with Fractional Laplacian Operators, while the second one by the MIUR National Research Project Nonlinear Elliptic Problems in the Study of Vortices and Related Topics and the FIRB project A\&B (Analysis and Beyond). Both the authors were supported by the ERC grant $\varepsilon$ (Elliptic Pde's and Symmetry of Interfaces and Layers for Odd Nonlinearities). 


\section{COnTEnts}

1. Introduction

2. Perron method and existence theory for viscosity solutions 137

3. Regularity theory for weak solutions

3.1. Maximum principle for weak solutions

3.2. Proof of Theorem 2

4. Proof of Theorem 1

5. On the boundedness of weak solutions

5.1. Proof of Proposition 9

\section{Introduction}

In the literature, different notions of solutions are taken into account when dealing with elliptic equations, such as, for instance, the weak (also called distributional, or variational, or energy) solutions (i.e. the solutions that belong to a suitable Sobolev space and satisfy the equation in a distributional sense, when integrated against a suitable set of test functions) and the viscosity solutions (i.e. all the smooth functions that touch either from above or below the continuous solution are required to be either subsolutions or supersolutions).

In this note, we point out that, for the fractional Laplace equation, the notion of weak solution implies the one of viscosity solution.

In what follows we take $\Omega$ to be a bounded, open subset of $\mathbb{R}^{n}$ with $C^{2}$-boundary and $s \in(0,1)$, and we consider the fractional boundary value problem

$$
\begin{cases}(-\Delta)^{s} u=f & \text { in } \Omega \\ u=g & \text { in } \mathbb{R}^{n} \backslash \Omega,\end{cases}
$$

where $f$ and $g$ are sufficiently smooth. The operator $(-\Delta)^{s}$ is the fractional Laplacian, that may be defined as

$$
(-\Delta)^{s} u(x):=c(n, s) \int_{\mathbb{R}^{n}} \frac{2 u(x)-u(x+y)-u(x-y)}{|y|^{n+2 s}} d y,
$$

for a suitable normalizing constant $c(n, s)>0$ (see, e.g., $[\mathbf{6}, \mathbf{9}, \mathbf{1 5}]$ for the basic theory of the fractional Laplacian and for the associated fractional Sobolev spaces). Of course, a more general framework could be considered, but we focus on problem (1.1) under the more basic assumptions for the sake of concreteness. 
The definition in (1.2) is well posed if $u$ is smooth enough and it does not grow too much at infinity (for instance if $u \in C^{2}\left(\mathbb{R}^{n}\right) \cap L^{\infty}\left(\mathbb{R}^{n}\right)$ ). On the other hand, it is convenient to consider weak formulations of (1.1), both to construct solutions using variational methods and to develop a regularity theory based on comparison principles.

These two different scopes indeed reflect two different attitudes and methodologies (namely test functions and energy estimates versus touching the solution with a barrier), and they are reminiscent of the dichotomy between operators in divergence and non-divergence form in the classical elliptic framework. These two different points of view naturally translate into two different notions of solutions, i.e. weak versus viscosity ones.

As a matter of fact, in the recent literature, some interest has been developed for weak solutions of (1.1), that is for functions $u \in \mathbb{H}^{s}(\Omega) \cap$ $L^{\infty}\left(\mathbb{R}^{n}\right)$ that satisfy

$$
\left\{\begin{array}{l}
c(n, s) \int_{\mathbb{R}^{2 n}} \frac{(u(x)-u(y))(\varphi(x)-\varphi(y))}{|x-y|^{n+2 s}} d x d y \\
\quad=\int_{\mathbb{R}^{n}} f(x) \varphi(x) d x \text { for any } \varphi \in \mathbb{H}_{0}^{s}(\Omega), \\
u=g \text { a.e. in } \mathbb{R}^{n} \backslash \Omega .
\end{array}\right.
$$

Here we denote by $\mathbb{R}^{2 n}:=\mathbb{R}^{n} \times \mathbb{R}^{n}$ and by $\mathbb{H}^{s}(\Omega)$ the set of all measurable functions $u: \mathbb{R}^{n} \rightarrow \mathbb{R}$ such that $u \in L^{2}(\Omega)$ and

$$
\int_{\mathbb{R}^{2 n} \backslash\left(\mathbb{R}^{n} \backslash \Omega\right)^{2}} \frac{|u(x)-u(y)|^{2}}{|x-y|^{n+2 s}} d x d y<+\infty .
$$

We also denoted by $\mathbb{H}_{0}^{s}(\Omega)$ the functions in $\mathbb{H}^{s}(\Omega)$ which vanish outside $\Omega$. The notation of $\mathbb{H}^{s}(\Omega)$ is of course reminiscent of the one for the classical Sobolev space $H^{s}(\Omega)$, see, e.g., [6]. We remark that $\mathbb{H}^{s}(\Omega) \subsetneq H^{s}(\Omega)$ since the domain of integration in (1.4) is larger than $\Omega \times \Omega$. On the other hand, such domain of integration may be equivalently replaced by the whole of $\mathbb{R}^{2 n}$ if $u$ vanishes outside $\Omega$, therefore $\mathbb{H}_{0}^{s}(\Omega)$ is the set of all functions in $H^{s}\left(\mathbb{R}^{n}\right)$ that vanish outside $\Omega$, hence $\mathbb{H}_{0}^{s}(\Omega)=H_{0}^{s}(\Omega)$.

The interest of these weak solutions mainly comes from the calculus of variations, since the expression in (1.3) arises from the Euler-Lagrange equation of a fractional type energy functional (see, e.g., $[\mathbf{1 2}, \mathbf{1 4}]$ and references therein).

On the other hand, when dealing with comparison principles, it is also natural to consider viscosity solutions of (1.1) (see, e.g., [15]). For 
this we recall that $u \in C\left(\mathbb{R}^{n}\right)$ is a viscosity subsolution (more generally, as in [4], one can define the notion of viscosity subsolution and supersolution for semicontinuous functions, so that this class is closed under sup and inf) for (1.1) if for any open set $U \subset \Omega$, any $x_{o} \in U$ and any $\phi \in C^{2}(U)$ such that $\phi\left(x_{o}\right)=u\left(x_{o}\right)$ and $\phi \geqslant u$ in $U$, if we let

$$
v(x):= \begin{cases}\phi(x) & \text { in } U \\ u(x) & \text { outside } U,\end{cases}
$$

we have that $-(-\Delta)^{s} v\left(x_{o}\right) \geqslant-f\left(x_{o}\right)$. Similarly, we say that $u \in$ $C\left(\mathbb{R}^{n}\right)$ is a viscosity supersolution for (1.1) if for any $\psi \in C^{2}(U)$ such that $\psi\left(x_{o}\right)=u\left(x_{o}\right)$ and $\psi \leqslant u$ in $U$, if we let

$$
w(x):= \begin{cases}\psi(x) & \text { in } U \\ u(x) & \text { outside } U\end{cases}
$$

we have that $-(-\Delta)^{s} w\left(x_{o}\right) \leqslant-f\left(x_{o}\right)$. Then, $u$ is a viscosity solution of problem (1.1) if it is both a viscosity subsolution and a viscosity supersolution.

Aim of this paper is to prove a relation between weak and viscosity solutions of problem (1.1). With respect to this, the main observation that we make in this note is the following one:

Theorem 1. Let $f \in C\left(\mathbb{R}^{n}\right)$ and $g \in C\left(\mathbb{R}^{n}\right) \cap L^{\infty}\left(\mathbb{R}^{n}\right)$. Let $u \in \mathbb{H}^{s}(\Omega) \cap$ $L^{\infty}\left(\mathbb{R}^{n}\right)$ be a weak solution of (1.1). Then, $u$ is a viscosity solution of $(1.1)$.

The converse of Theorem 1 holds true if $f$ is smooth enough thanks to the regularity theory for viscosity solutions, see [10]. (After a first draft of this note was completed, we have received the very nice paper $[\mathbf{1 1}]$, which contains, among others, a result closely related to Theorems 1 and 2 obtained with different proofs.)

In order to prove Theorem 1 we first need to show that every weak solution of (1.1) is continuous in $\mathbb{R}^{n}$, as stated in the following theorem:

Theorem 2. Let $f \in L^{\infty}(\Omega)$ and let $u \in \mathbb{H}^{s}(\Omega) \cap L^{\infty}\left(\mathbb{R}^{n}\right) \cap C\left(\mathbb{R}^{n} \backslash \Omega\right)$ be a weak solution of

$$
(-\Delta)^{s} u=f \quad \text { in } \quad \Omega \text {. }
$$

Then, $u \in C\left(\mathbb{R}^{n}\right)$.

For this, we first give an interior regularity result for weak solutions (see Proposition 5 for the details) and, later, we show that the weak solutions of our problem are continuous up to the boundary (hence in the whole $\mathbb{R}^{n}$ ), if so are outside the domain where the problem is set. 
As a byproduct of Theorem 2, [13, Proposition 9-b) and Appendix A] and [14, Proposition 4], in Corollary 8 we show that the first eigenfunction of $(-\Delta)^{s}$ with Dirichlet data is strictly positive in $\Omega$.

We would like to note that in the definition of weak solution and in all the previous results we assume that the weak solutions of problem (1.1) are in $L^{\infty}(\Omega)$. We do this only for the sake of clarity and concreteness. In fact, this condition can be removed. Indeed, in Proposition 9 we show that every weak solution of $(1.1)$ is in $L^{\infty}(\Omega)$, provided the data are smooth enough.

The paper is organized as follows. In Section 2 we recall the Perron method and the existence theory of viscosity solutions for fractional boundary value problems. Then, in Section 3 we develop the regularity theory for weak solutions, by establishing continuity up to the boundary via a suitable maximum principle for weak solutions, and so proving Theorem 2: with this we will be able to complete the proof of Theorem 1 in Section 4. Finally, in Section 5 we point out that weak solutions are automatically bounded.

\section{Perron method and existence theory for viscosity solutions}

Here we recall that the classical Perron method (see, e.g., [8]) can be suitably adapted to fractional problems, giving an existence theory in the viscosity setting. One way to construct solutions somehow explicitly consists in using the fractional Poisson kernel in the ball in order to reconstruct harmonic functions from their values outside (see, e.g., $[\mathbf{9}]$ ) and then taking the supremum of all possible subsolutions. The notion of half relaxed limit makes this construction compatible with the notion of viscosity solutions. Suitable barriers are needed to check that the boundary data are attained, as in [5]: this is more complicated than in the classical framework of the Laplacian since the fundamental solution is not a barrier, because an inequality in the whole complement is necessary for the comparison principle in this case.

For instance, as a particular case of $[\mathbf{1}$, Theorem 1] we have the following result:

Proposition 3. Let $\Omega$ be open, bounded and with $C^{2}$-boundary and let $g \in C\left(\mathbb{R}^{n}\right) \cap L^{\infty}\left(\mathbb{R}^{n}\right)$. Then, there exists a viscosity solution $u \in C\left(\mathbb{R}^{n}\right) \cap$ $L^{\infty}\left(\mathbb{R}^{n}\right)$ of the problem

$$
\begin{cases}(-\Delta)^{s} u=0 & \text { in } \Omega \\ u=g & \text { in } \mathbb{R}^{n} \backslash \Omega .\end{cases}
$$


As a consequence of Proposition 3 we have the following existence result:

Corollary 4. Let $\Omega$ be open, bounded and with $C^{2}$-boundary. Let $f \in$ $C^{2}\left(\mathbb{R}^{n}\right)$ and $g \in C\left(\mathbb{R}^{n}\right) \cap L^{\infty}\left(\mathbb{R}^{n}\right)$. Then, there exists a viscosity solution $u \in C\left(\mathbb{R}^{n}\right) \cap L^{\infty}\left(\mathbb{R}^{n}\right)$ of the problem

$$
\begin{cases}(-\Delta)^{s} u=f & \text { in } \Omega \\ u=g & \text { in } \mathbb{R}^{n} \backslash \Omega .\end{cases}
$$

Proof: We may modify $f$ outside $\Omega$ in such a way that $f$ is compactly supported in some $B_{R}, R>0$, and therefore bounded and uniformly continuous. We take $\Gamma(x):=c(n, s)|x|^{2 s-n}$ the fundamental solution of the fractional Laplacian (see, e.g., $[\mathbf{9}$, p. 44] and [3, Subsection 2.2] for the basic properties of fractional fundamental solutions), that is $(-\Delta)^{s} \Gamma=$ $\delta_{0}$, where $\delta_{0}$ is the Dirac delta function in 0 .

Let $\tilde{g}:=g-\Gamma * f$. Notice that

$$
\Gamma * f(x)=c(n, s) \int_{B_{R}(x)}|y|^{2 s-n} f(x-y) d y,
$$

thanks to our further assumptions on $f$. Since $s<1$

$$
\int_{B_{R}(x)}|y|^{2 s-n} d y<+\infty
$$

and so we obtain that $\Gamma * f \in C\left(\mathbb{R}^{n}\right) \cap L^{\infty}\left(\mathbb{R}^{n}\right)$, again by the assumptions on $f$. Accordingly, $\tilde{g} \in C\left(\mathbb{R}^{n}\right) \cap L^{\infty}\left(\mathbb{R}^{n}\right)$, so we may apply Proposition 3 and obtain a viscosity solution $\tilde{u}$ of $(-\Delta)^{s} \tilde{u}=0$ in $\Omega$ and $\tilde{u}=\tilde{g}$ outside $\Omega$.

Also $\Gamma * f \in C^{2}\left(\mathbb{R}^{n}\right)$ and it solves

$$
(-\Delta)^{s}(\Gamma * f)=\left((-\Delta)^{s} \Gamma\right) * f=f .
$$

Let $u:=\tilde{u}+\Gamma * f$. Then, $u$ solves (2.1) and this ends the proof of Corollary 4.

\section{Regularity theory for weak solutions}

In this section we give some regularity results for weak solutions of problem (1.1). First of all, next proposition deals with the interior regularity theory for weak solutions: 
Proposition 5. Let $f \in L^{\infty}\left(B_{1}\right)$ and let $u \in \mathbb{H}^{s}\left(B_{3}\right) \cap L^{\infty}\left(\mathbb{R}^{n}\right)$ be a weak solution of

$$
(-\Delta)^{s} u=f \quad \text { in } \quad B_{1} .
$$

Then, $u \in C^{\alpha}\left(B_{1 / 4}\right)$ for any $0<\alpha<\min \{2 s, 1\}$ and

$$
\|u\|_{C^{\alpha}\left(B_{1 / 4}\right)} \leqslant C\left(\|u\|_{L^{\infty}\left(\mathbb{R}^{n}\right)}+\|f\|_{L^{\infty}\left(B_{1}\right)}\right),
$$

for a suitable $C>0$ depending on $n, s$ and $\alpha$.

Proof: The case of smooth solutions was considered in [7], so it is enough to reduce to this case by a standard convolution argument. Namely, we take $\rho \in C_{0}^{\infty}\left(B_{1} ;[0,1]\right)$ and we consider the mollifier $\rho_{\varepsilon}(x):=\varepsilon^{-n} \rho(x / \varepsilon)$, $\varepsilon>0$. We define $u_{\varepsilon}:=u * \rho_{\varepsilon}$ and $f_{\varepsilon}:=f * \rho_{\varepsilon}$.

Of course, $u_{\varepsilon}$ is a smooth function, by construction. Let us show that $u_{\varepsilon}$ solves the following equation

$$
(-\Delta)^{s} u_{\varepsilon}=f_{\varepsilon} \quad \text { in } \quad B_{1 / 2} .
$$

For this, first we observe that for any test function $\phi$ supported in $B_{1 / 2}$

$$
\begin{aligned}
& \int_{\mathbb{R}^{n}}\left[\int_{\mathbb{R}^{2 n}} \frac{|u(x+z)-u(y+z)||\phi(x)-\phi(y)| \rho_{\varepsilon}(z)}{|x-y|^{n+2 s}} d x d y\right] d z \\
& \quad=\int_{B_{\varepsilon}}\left[\int_{Q\left(B_{1}\right)} \frac{|u(x+z)-u(y+z)||\phi(x)-\phi(y)| \rho_{\varepsilon}(z)}{|x-y|^{n+2 s}} d x d y\right] d z \\
& \quad \leqslant \frac{\varepsilon^{-n}}{2} \int_{B_{\varepsilon}}\left[\int_{Q\left(B_{2}\right)} \frac{|u(x)-u(y)|^{2}}{|x-y|^{n+2 s}} d x d y+\int_{Q\left(B_{1}\right)} \frac{|\phi(x)-\phi(y)|^{2}}{|x-y|^{n+2 s}} d x d y\right] d z \\
& \quad<+\infty,
\end{aligned}
$$

since $u \in \mathbb{H}^{s}\left(B_{3}\right) \cap L^{\infty}\left(\mathbb{R}^{n}\right)$. Here we used the notation

$$
Q\left(B_{r}\right):=\mathbb{R}^{2 n} \backslash\left(\left(\mathbb{R}^{n} \backslash B_{r}\right) \times\left(\mathbb{R}^{n} \backslash B_{r}\right)\right), \quad r>0 .
$$

Hence, by Tonelli's Theorem the function

$$
\mathbb{R}^{2 n} \times \mathbb{R}^{n} \ni(x, y, z) \mapsto \frac{(u(x+z)-u(y+z))(\phi(x)-\phi(y)) \rho_{\varepsilon}(z)}{|x-y|^{n+2 s}}
$$


belongs to $L^{1}\left(\mathbb{R}^{2 n} \times \mathbb{R}^{n}\right)$. Then, by Fubini's Theorem

$$
\begin{aligned}
\int_{\mathbb{R}^{n}} & {\left[\int_{\mathbb{R}^{2 n}} \frac{(u(x+z)-u(y+z))(\phi(x)-\phi(y)) \rho_{\varepsilon}(z)}{|x-y|^{n+2 s}} d x d y\right] d z } \\
& =\int_{\mathbb{R}^{2 n}}\left[\int_{\mathbb{R}^{n}} \frac{(u(x+z)-u(y+z))(\phi(x)-\phi(y)) \rho_{\varepsilon}(z)}{|x-y|^{n+2 s}} d z\right] d x d y \\
& =\int_{\mathbb{R}^{2 n}} \frac{\left(u_{\varepsilon}(x)-u_{\varepsilon}(y)\right)(\phi(x)-\phi(y))}{|x-y|^{n+2 s}} d x d y,
\end{aligned}
$$

thanks to the definition of $u_{\epsilon}$.

Therefore, we may use Fubini's Theorem and obtain that for any $\phi \in C_{0}^{\infty}\left(B_{1 / 2}\right)$

$$
\begin{aligned}
\int_{\mathbb{R}^{n}} f_{\varepsilon}(x) \phi(x) d x \\
=\int_{\mathbb{R}^{n}}\left[\int_{\mathbb{R}^{n}} f(x+z) \phi(x) \rho_{\varepsilon}(z) d x\right] d z \\
=\int_{B_{\varepsilon}}\left[\int_{\mathbb{R}^{n}} f(\tilde{x}) \phi(\tilde{x}-z) \rho_{\varepsilon}(z) d \tilde{x}\right] d z \\
=\int_{B_{\varepsilon}}\left[\int_{\mathbb{R}^{2 n}} \frac{(u(\tilde{x})-u(\tilde{y}))(\phi(\tilde{x}-z)-\phi(\tilde{y}-z)) \rho_{\varepsilon}(z)}{|\tilde{x}-\tilde{y}|^{n+2 s}} d \tilde{x} d \tilde{y}\right] d z \\
=\int_{\mathbb{R}^{n}}\left[\int_{\mathbb{R}^{2 n}} \frac{(u(x+z)-u(y+z))(\phi(x)-\phi(y)) \rho_{\varepsilon}(z)}{|x-y|^{n+2 s}} d x d y\right] d z \\
=\int_{\mathbb{R}^{2 n}} \frac{\left(u_{\varepsilon}(x)-u_{\varepsilon}(y)\right)(\phi(x)-\phi(y))}{|x-y|^{n+2 s}} d x d y,
\end{aligned}
$$

thanks to the fact that $u$ is a weak solution of (3.1). This means that $u_{\varepsilon}$ is a smooth solution of (3.2). 
As a consequence of this, we may apply [7, Corollary 4.3] and obtain that $u_{\varepsilon} \in C^{\alpha}\left(B_{1 / 4}\right)$ for any $0<\alpha<\min \{2 s, 1\}$ and

$$
\left\|u_{\varepsilon}\right\|_{C^{\alpha}\left(B_{1 / 4}\right)} \leqslant C\left(\left\|u_{\varepsilon}\right\|_{L^{\infty}\left(\mathbb{R}^{n}\right)}+\left\|f_{\varepsilon}\right\|_{L^{\infty}\left(B_{1}\right)}\right),
$$

for a suitable $C>0$ depending on $n, s$ and $\alpha$. Hence, the desired result follows by sending $\varepsilon \searrow 0$.

3.1. Maximum principle for weak solutions. Here we prove a maximum principle for weak solutions in $\mathbb{H}^{s}$ which is the natural extension of the classical one in $H^{1}$ :

Lemma 6. Let $v \in \mathbb{H}^{s}(\Omega)$ satisfy $(-\Delta)^{s} v \leqslant 0$ in the weak sense, with $v \leqslant 0$ in $\mathbb{R}^{n} \backslash \Omega$. Then $v \leqslant 0$ in $\mathbb{R}^{n}$.

Proof: By assumption $v^{+}=0$ in $\mathbb{R}^{n} \backslash \Omega$, hence $v^{+} \in \mathbb{H}_{0}^{s}(\Omega)$ and so we can use it as a test function in the weak formulation of the problem. Therefore, setting

$$
\langle\phi, \psi\rangle_{s}:=c(n, s) \int_{\mathbb{R}^{2 n}} \frac{(\phi(x)-\phi(y))(\psi(x)-\psi(y))}{|x-y|^{n+2 s}} d x d y
$$

and

$$
\|\phi\|_{s}:=\sqrt{\langle\phi, \phi\rangle_{s}}
$$

from the problem we obtain that

$$
\left\langle v^{+}, v\right\rangle_{s} \leqslant 0 .
$$

As a consequence of this, writing $v^{-}:=v^{+}-v \geqslant 0$, and using that $v^{+}(x) v^{-}(x)=0$ and $v^{+}(x) v^{-}(y) \geqslant 0$ a.e. $x, y \in \mathbb{R}^{n}$, we get

$$
\begin{aligned}
0 \geqslant\left\langle v^{+}, v\right\rangle_{s} & =\left\langle v^{+}, v^{+}-v^{-}\right\rangle_{s} \\
& =\left\|v^{+}\right\|_{s}^{2}-\left\langle v^{+}, v^{-}\right\rangle_{s} \\
& =\left\|v^{+}\right\|_{s}^{2}-\int_{\mathbb{R}^{2 n}} \frac{\left(v^{+}(x)-v^{+}(y)\right)\left(v^{-}(x)-v^{-}(y)\right)}{|x-y|^{n+2 s}} d x d y \\
& =\left\|v^{+}\right\|_{s}^{2}+\int_{\mathbb{R}^{2 n}} \frac{\left(v^{+}(x) v^{-}(y)+v^{-}(x) v^{+}(y)\right)}{|x-y|^{n+2 s}} d x d y \\
& \geqslant\left\|v^{+}\right\|_{s}^{2} .
\end{aligned}
$$

Therefore, $v^{+}=0$ in $\mathbb{R}^{n}$ and so the claim is proved. 
Now we are ready to show that the weak solutions of the fractional Laplace equation are continuous up to the boundary (hence in the whole of the space), if so are outside the domain where the problem is set. With respect to this our result is stated in Theorem 2. Here below we provide a proof of it.

3.2. Proof of Theorem 2. The idea of the proof is to consider a smooth neighborhood $\Omega_{\varepsilon}$ of $\Omega$ and a continuous function $\beta_{\varepsilon}$ that agrees with $u$ outside $\Omega_{\varepsilon}$ and with $(-\Delta)^{s} \beta_{\varepsilon}$ very large (by the smoothness of $\beta_{\varepsilon}$ in $\Omega_{\varepsilon}$, the notion of viscosity and weak solution are the same for it). By possibly translating $\beta_{\varepsilon}$ a little bit up, one checks that $\beta_{\varepsilon}$ must be above $u$. Then, the continuity of $\beta_{\varepsilon}$ gives a control from above of the values of $u$ near $\partial \Omega$. By a reverse argument, one obtains a control from below, and hence the continuity of $u$ near $\partial \Omega$. Below are the details of this argument.

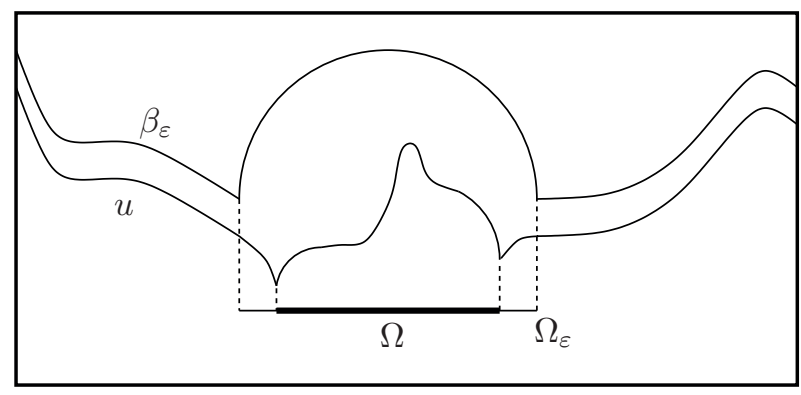

Figure 1. The barrier $\beta_{\varepsilon}$ which bounds $u$ from above.

We argue by contradiction, by supposing that there exist $x_{o} \in \mathbb{R}^{n}$ and a sequence $x_{j}$ in $\mathbb{R}^{n}$ such that

$$
\lim _{j \rightarrow+\infty} x_{j}=x_{o} \quad \text { but }\left|u\left(x_{j}\right)-u\left(x_{o}\right)\right| \geqslant a
$$

for some $a>0$. For definiteness, we suppose that

$$
u\left(x_{j}\right)-u\left(x_{o}\right) \geqslant a
$$

since the case $u\left(x_{o}\right)-u\left(x_{j}\right) \geqslant a$ can be treated in a similar way.

First of all, we claim that

$$
x_{o} \in \partial \Omega \text {. }
$$

Indeed, $x_{o} \in \bar{\Omega}$, since $u$ is continuous in the open set $\mathbb{R}^{n} \backslash \bar{\Omega}$, by assumption. Moreover, it cannot be that $x_{o}$ lies in the interior of $\Omega$, because $u$ is 
also continuous in any domain compactly contained in $\Omega$, thanks to the local regularity theory stated in Proposition 5. This proves (3.4).

Now let $\varepsilon \in(0,2]$ and let $\Omega_{\varepsilon}$ be a smooth $\varepsilon$-neighborhood of $\Omega$, that is a set with $C^{2}$-boundary and such that

$$
\bigcup_{x \in \Omega} B_{\varepsilon / 2}(x) \subseteq \Omega_{\varepsilon} \subseteq \bigcup_{x \in \Omega} B_{\varepsilon}(x) .
$$

Moreover, let $\beta_{\varepsilon}$ be the viscosity solution of

$$
\begin{cases}(-\Delta)^{s} \beta_{\varepsilon}=M & \text { in } \Omega_{\varepsilon} \\ \beta_{\varepsilon}=u & \text { in } \mathbb{R}^{n} \backslash \Omega_{\varepsilon},\end{cases}
$$

where $M:=\|f\|_{L^{\infty}(\Omega)}$. The existence of such a viscosity solution is warranted by Corollary 4 and by the regularity assumptions on $u$. To be precise, in order to use Corollary 4 to obtain the desired solution $\beta_{\varepsilon}$ in (3.5), one needs to extend the datum $u$ continuously inside $\Omega_{\varepsilon}$. This can be accomplished by taking $\phi_{\varepsilon} \in C^{\infty}\left(\mathbb{R}^{n}\right)$ such that $\phi_{\varepsilon}=1$ in $\mathbb{R}^{n} \backslash$ $\Omega_{\varepsilon / 2}$ and $\phi_{\varepsilon}=0$ in $\Omega_{\varepsilon / 4}$. Then one defines $g_{\varepsilon}:=\phi_{\varepsilon} u+\left(1-\phi_{\varepsilon}\right)$. By construction $g_{\varepsilon} \in C\left(\mathbb{R}^{n}\right)$, so Corollary 4 gives the existence of a viscosity solution $\beta_{\varepsilon}$ of $(-\Delta)^{s} \beta_{\varepsilon}=M$ in $\Omega_{\varepsilon}$, with $\beta_{\varepsilon}=g_{\varepsilon}=u$ in $\mathbb{R}^{n} \backslash \Omega_{\varepsilon}$.

Note that $\beta_{\varepsilon} \in C\left(\bar{\Omega}_{\varepsilon}\right)$ (again by Corollary 4) with modulus of continuity of $\beta_{\varepsilon}$ in $\Omega_{\varepsilon}$ independent of $\varepsilon$ : see for instance [5, Theorem 32] for the continuity up to the boundary. (We stress that the modulus of continuity of $\beta_{\varepsilon}$ may depend on the $C^{2}$-regularity of $\partial \Omega$. As a technical remark, we also notice that the barrier used in [5] does not belong to a good energy space like $\mathbb{H}^{s}(\Omega)$ and therefore it cannot be used to prove directly the boundary continuity of weak solutions.) Also, by interior regularity (see $[\mathbf{2}$, Theorem 5$]$ ), we have that $\beta_{\varepsilon}$ is as smooth as we like in any domain compactly contained in $\Omega_{\varepsilon}$ (of course the smooth norm involved depends on the distance of the domain from $\left.\partial \Omega_{\varepsilon}\right)$. All in all, we obtain that

$$
\begin{array}{ll}
(-\Delta)^{s} \beta_{\varepsilon}=M & \text { in } \Omega_{\varepsilon} \text { in the viscosity sense, } \\
\beta_{\varepsilon}=u & \text { in } \mathbb{R}^{n} \backslash \Omega_{\varepsilon}, \quad \text { and } \\
\beta_{\varepsilon} \in C^{2}(\bar{\Omega}) \cap C\left(\bar{\Omega}_{\varepsilon}\right) &
\end{array}
$$

with modulus of continuity $\rho$ of $\beta_{\varepsilon}$ in $\Omega_{\varepsilon}$, defined as

$$
\rho(t)=\sup _{\substack{x, y \in \Omega_{\varepsilon} \\|x-y|<t}}\left|\beta_{\varepsilon}(x)-\beta_{\varepsilon}(y)\right| .
$$

Note that $\rho$ is independent of $\varepsilon$, while $\left\|\beta_{\varepsilon}\right\|_{C^{2}(\bar{\Omega})}$ may depend on $\varepsilon$. 
Now, let also $\sigma$ be the modulus of continuity of $u$ in the compact set $\bar{\Omega}_{2} \backslash \Omega \subset \mathbb{R}^{n} \backslash \Omega$ (of course $\Omega_{2}$ is simply $\Omega_{\varepsilon}$ for $\varepsilon=2$ ). That is

$$
\sigma(t)=\sup _{\substack{x, y \in \bar{\Omega}_{2} \backslash \Omega \\|x-y|<t}}|u(x)-u(y)| .
$$

We set

$$
\eta_{\varepsilon}:=\varepsilon+\rho(\varepsilon)+\sigma(\varepsilon) .
$$

First of all, we point out that, thanks to the continuity properties of $\beta_{\varepsilon}$ and $u$,

$$
\lim _{\varepsilon \searrow 0} \eta_{\varepsilon}=0
$$

Moreover, we claim that

$$
\beta_{\varepsilon}+\eta_{\varepsilon}>u \text { in } \mathbb{R}^{n} \backslash \Omega \text {. }
$$

To check this, let $x \in \mathbb{R}^{n} \backslash \Omega$. First, if $x \in \mathbb{R}^{n} \backslash \Omega_{\varepsilon}$, then $\beta_{\varepsilon}(x)=u(x)$, due to (3.6), and so (3.9) holds true. If, on the other hand, $x \in \Omega_{\varepsilon} \backslash \Omega$, then there exists $y \in \partial \Omega_{\varepsilon} \subseteq \mathbb{R}^{n} \backslash \Omega_{\varepsilon}$ such that $|x-y| \leqslant \varepsilon$. Therefore $\beta_{\varepsilon}(y)=u(y),\left|\beta_{\varepsilon}(x)-\beta_{\varepsilon}(y)\right| \leqslant \rho(\varepsilon)$ and $|u(x)-u(y)| \leqslant \sigma(\varepsilon)$. As a consequence,

$\beta_{\varepsilon}(x)-u(x) \geqslant \beta_{\varepsilon}(y)-u(y)-(\rho(\varepsilon)+\sigma(\varepsilon))=-(\rho(\varepsilon)+\sigma(\varepsilon))=-\eta_{\varepsilon}+\varepsilon>-\eta_{\varepsilon}$, which proves (3.9).

Now, let $v_{\varepsilon}:=u-\beta_{\varepsilon}-\eta_{\varepsilon}$. We claim that

$$
v_{\varepsilon} \leqslant 0 \text { in } \mathbb{R}^{n} .
$$

For this, note that, by (3.6) and the assumptions on $u$, the function $v_{\varepsilon}$ is a weak solution of

$$
(-\Delta)^{s} v_{\varepsilon}=f-M \leqslant 0 \text { in } \Omega,
$$

thanks to the choice of $M$. Moreover, by (3.9), we know that $v_{\varepsilon} \leqslant 0$ in $\mathbb{R}^{n} \backslash \Omega$. Also, $v_{\varepsilon} \in \mathbb{H}^{s}(\Omega)$ since so are $u$ and $\beta_{\varepsilon}$, by the assumptions on $u$ and (3.6). Accordingly, we can use Lemma 6 and obtain (3.10).

This gives that $u \leqslant \beta_{\varepsilon}+\eta_{\varepsilon}$ in $\mathbb{R}^{n}$ by the definition of $v_{\varepsilon}$. Thus, from this, (3.3) and the continuity of $\beta_{\varepsilon}$, we see that, fixed $\varepsilon \in(0,2)$, for large $j$,

$$
\begin{aligned}
a+u\left(x_{o}\right) & \leqslant u\left(x_{j}\right) \\
& \leqslant \beta_{\varepsilon}\left(x_{j}\right)+\eta_{\varepsilon} \\
& \leqslant \beta_{\varepsilon}\left(x_{o}\right)+\rho\left(\left|x_{j}-x_{o}\right|\right)+\eta_{\varepsilon} \\
& \leqslant \beta_{\varepsilon}\left(x_{o}\right)+2 \eta_{\varepsilon},
\end{aligned}
$$


since $\rho\left(\left|x_{j}-x_{o}\right|\right)=\eta_{\left|x_{j}-x_{o}\right|}-\sigma\left(\left|x_{j}-x_{o}\right|\right)-\left|x_{j}-x_{o}\right|<\eta_{\left|x_{j}-x_{o}\right|}<\eta_{\varepsilon}$ by (3.7) and the choice of $x_{j}$.

Moreover, recalling (3.4), we know that there exists $y_{\varepsilon} \in \mathbb{R}^{n} \backslash \Omega_{\varepsilon}$ such that $\left|y_{\varepsilon}-x_{o}\right| \leqslant \varepsilon$. Notice that $\beta_{\varepsilon}\left(y_{\varepsilon}\right)=u\left(y_{\varepsilon}\right)$ by (3.6). Therefore, (3.11) gives

$$
\begin{aligned}
a+u\left(x_{o}\right) & \leqslant \beta_{\varepsilon}\left(x_{o}\right)+2 \eta_{\varepsilon} \\
& =u\left(y_{\varepsilon}\right)+\beta_{\varepsilon}\left(x_{o}\right)-\beta_{\varepsilon}\left(y_{\varepsilon}\right)+2 \eta_{\varepsilon} \\
& \leqslant u\left(y_{\varepsilon}\right)+3 \eta_{\varepsilon},
\end{aligned}
$$

being

$$
\begin{aligned}
\left|\beta_{\varepsilon}\left(x_{o}\right)-\beta_{\varepsilon}\left(y_{\varepsilon}\right)\right| & \leqslant \rho\left(\left|x_{o}-y_{\varepsilon}\right|\right) \\
= & \eta_{\left|x_{o}-y_{\varepsilon}\right|}-\sigma\left(\left|x_{o}-y_{\varepsilon}\right|\right)-\left|x_{o}-y_{\varepsilon}\right|<\eta_{\left|x_{o}-y_{\varepsilon}\right|}<\eta_{\varepsilon} .
\end{aligned}
$$

Also, $y_{\varepsilon}, x_{o} \in \Omega_{2} \backslash \Omega$, since, by construction, $\Omega_{\varepsilon} \subset \Omega_{2}$ (being $\varepsilon<2$ ) and $\left|y_{\varepsilon}-x_{o}\right| \leqslant \varepsilon$. Thus, being $u \in C\left(\mathbb{R}^{n} \backslash \Omega\right)$

$$
u\left(y_{\varepsilon}\right)-u\left(x_{o}\right) \leqslant \sigma\left(\left|y_{\varepsilon}-x_{o}\right|\right) \leqslant \sigma(\varepsilon) \leqslant \eta_{\varepsilon},
$$

thanks to the fact that $\sigma(\varepsilon)=\eta_{\varepsilon}-\rho(\varepsilon)-\varepsilon<\eta_{\varepsilon}$. Thus, this and (3.12) imply that

$$
a+u\left(x_{o}\right) \leqslant u\left(y_{\varepsilon}\right)+3 \eta_{\varepsilon} \leqslant u\left(y_{\varepsilon}\right)-u\left(x_{o}\right)+u\left(x_{o}\right)+3 \eta_{\varepsilon} \leqslant u\left(x_{o}\right)+4 \eta_{\varepsilon},
$$

that is

$$
a \leqslant 4 \eta_{\varepsilon}
$$

By taking the limit as $\varepsilon \searrow 0$ and recalling (3.8) we obtain $a \leqslant 0$, which is against our assumption. Thus, $u \in C\left(\mathbb{R}^{n}\right)$ and this concludes the proof of Theorem 2 .

\section{Proof of Theorem 1}

By extending $f$ in a bounded way outside $\Omega$, we may suppose that $f \in L^{\infty}\left(\mathbb{R}^{n}\right)$. Let $u$ be a weak solution of problem (1.1). Since $u=g$ a.e. in $\mathbb{R}^{n} \backslash \Omega$, then $u \in C\left(\mathbb{R}^{n} \backslash \Omega\right)$, thanks to the assumptions on $g$. Then, we can apply Theorem 2 and get that

$$
u \in C\left(\mathbb{R}^{n}\right) .
$$

Now, let us fix $x_{o} \in \Omega$. By convolution (as done in Proposition 5) and recalling the stability of viscosity solutions (see, e.g., [4, Lemma 4.5]) 
we may suppose that $u$ is also smooth in a small neighborhood $\Omega^{\prime}$ of $x_{o}$ and, from (1.3), for any $\varphi \in C_{0}^{\infty}\left(\Omega^{\prime}\right)$

$$
\begin{aligned}
c(n, s) \int_{\mathbb{R}^{2 n}} & \frac{(2 u(x)-u(x+Y)-u(x-Y)) \varphi(x)}{|Y|^{n+2 s}} d x d Y \\
& =c(n, s) \int_{\mathbb{R}^{2 n}} \frac{(u(x)-u(y))(\varphi(x)-\varphi(y))}{|x-y|^{n+2 s}} d x d y \\
& =\int_{\mathbb{R}^{n}} f(x) \varphi(x) d x .
\end{aligned}
$$

Therefore,

$$
c(n, s) \int_{\mathbb{R}^{n}} \frac{(2 u(x)-u(x+Y)-u(x-Y))}{|Y|^{n+2 s}} d Y=f(x)
$$

for almost any $x \in \Omega^{\prime}$ and, in fact, for any $x \in \Omega^{\prime}$, due to (4.1). In particular

$$
c(n, s) \int_{\mathbb{R}^{n}} \frac{\left(2 u\left(x_{o}\right)-u\left(x_{o}+Y\right)-u\left(x_{o}-Y\right)\right)}{|Y|^{n+2 s}} d Y=f\left(x_{o}\right) .
$$

Now, let us take $v$ as in (1.5): since $v \geqslant u$ in $\mathbb{R}^{n}$ and $v\left(x_{o}\right)=u\left(x_{o}\right)$, we obtain

$$
\begin{aligned}
(-\Delta)^{s} v\left(x_{o}\right) & =c(n, s) \int_{\mathbb{R}^{n}} \frac{\left(2 v\left(x_{o}\right)-v\left(x_{o}+Y\right)-v\left(x_{o}-Y\right)\right)}{|Y|^{n+2 s}} d Y \\
& \leqslant c(n, s) \int_{\mathbb{R}^{n}} \frac{\left(2 u\left(x_{o}\right)-u\left(x_{o}+Y\right)-u\left(x_{o}-Y\right)\right)}{|Y|^{n+2 s}} d Y \\
& =f\left(x_{o}\right) .
\end{aligned}
$$

This shows that $u$ is a viscosity subsolution of (1.1), and one can prove similarly that $u$ is a viscosity supersolution. This ends the proof of Theorem 1.

Remark 7. The result of Theorem 1 also holds when $f=f(x, u)$ for $f \in C\left(\mathbb{R}^{n+1}\right)$. Indeed, one sets $\tilde{f}(x):=f(x, u(x)) \in L^{\infty}\left(\mathbb{R}^{n+1}\right)$ and applies Theorem 2 to obtain that $u$ is continuous and therefore so is $\tilde{f}$ (in this case it is necessary to assume that $u$ is bounded from the beginning). Then, one may apply Theorem 1 (with $\tilde{f}$ in the place of $f$ ) and obtain that $u$ is a viscosity solution of (1.1). 
As a consequence of Theorem 2, we can prove the following result about the first eigenfunction of the operator $(-\Delta)^{s}$ :

Corollary 8. Let $\lambda_{1}$ be the first eigenvalue of the operator $(-\Delta)^{s}$ in $\Omega$ with Dirichlet boundary datum and $e_{1} \in \mathbb{H}_{0}^{s}(\Omega)$ be the corresponding eigenfunction, i.e.

$$
\begin{cases}(-\Delta)^{s} e_{1}=\lambda_{1} e_{1} & \text { in } \Omega \\ e_{1}=0 & \text { in } \mathbb{R}^{n} \backslash \Omega .\end{cases}
$$

Then, $e_{1}>0$ in $\Omega$.

Proof: By [14, Proposition 4] we know that $e_{1} \in L^{\infty}(\Omega)$. Hence, by Theorem 2 it is easily seen that $e_{1} \in C\left(\mathbb{R}^{n}\right)$.

Moreover, as a consequence of this and of [13, Proposition 9- $b$ )] we have that

$$
e_{1}(x) \geqslant 0 \quad \text { for any } \quad x \in \Omega \text {. }
$$

Also,

$$
(-\Delta)^{s} e_{1}(x) \geqslant 0 \text { for any } x \in \Omega,
$$

thank to the positivity of $\lambda_{1}$ (for this see [13, Proposition 9-a)]).

Assume by contradiction that there exists $\tilde{x} \in \Omega$ such that $e_{1}(\tilde{x})=0$. Then, by (1.2), (4.2) and (4.3) we get

$$
0 \leqslant(-\Delta)^{s} e_{1}(\tilde{x})=-c(n, s) \int_{\mathbb{R}^{n}} \frac{e_{1}(\tilde{x}+y)+e_{1}(\tilde{x}-y)}{|y|^{n+2 s}} d y \leqslant 0,
$$

so that, using also $(4.2), e_{1}(\tilde{x}+y)+e_{1}(\tilde{x}-y)=0$ in the whole of $\mathbb{R}^{n}$. Hence, by this and again (4.2) we get that $e_{1} \equiv 0$ in $\mathbb{R}^{n}$. Of course this is a contradiction, being $e_{1}$ an eigenfunction.

Hence, $e_{1}>0$ in $\Omega$ and this completes the proof of Corollary 8 .

\section{On the boundedness of weak solutions}

In this section we discuss the boundedness of weak solutions of problem (1.1).

In all the previous results of the present paper, for the sake of simplicity, we have focused on the case of bounded solutions of (1.1), i.e. on solutions $u \in L^{\infty}\left(\mathbb{R}^{n}\right)$. Here we show that, in fact, this assumption can be removed. Indeed, the boundedness of solutions may be proved directly using a barrier and the comparison principle for weak solutions (see the barrier $\beta_{\varepsilon}$ and the argument in Subsection 3.2). On the other hand, we present here a different argument of classical flavor which is suited also 
for more general fractional operators in divergence form. Namely, the following result holds true:

Proposition 9. Let $u \in \mathbb{H}^{s}(\Omega)$ be a weak solution of problem (1.1) (i.e., suppose that (1.3) holds), with $f \in C\left(\mathbb{R}^{n}\right)$ and $g \in C\left(\mathbb{R}^{n}\right) \cap L^{\infty}\left(\mathbb{R}^{n}\right)$. Then, $u \in L^{\infty}\left(\mathbb{R}^{n}\right)$.

For the proof of it, we single out some general energy estimates of fractional type, stated in the forthcoming Lemma 10 and Lemma 11.

Lemma 10. For any $v \in \mathbb{H}^{s}\left(\mathbb{R}^{n}\right)$

$$
(v(x)-v(y))\left(v^{+}(x)-v^{+}(y)\right) \geqslant\left|v^{+}(x)-v^{+}(y)\right|^{2}
$$

for any $x, y \in \mathbb{R}^{n}$.

Lemma 11. Let $f \in L^{2}(\Omega)$ and $v \in \mathbb{H}^{s}\left(\mathbb{R}^{n}\right)$ with $v=0$ in $\mathbb{R}^{n} \backslash \Omega$. Suppose that $(-\Delta)^{s} v=f$ in $\Omega$ in the weak sense and let $w:=v^{+}$. Then

$$
\int_{\mathbb{R}^{2 n}} \frac{|w(x)-w(y)|^{2}}{|x-y|^{n+2 s}} d x d y \leqslant \int_{\Omega} f(x) w(x) d x .
$$

Note that in the classical case, (5.1) boils down to $\nabla v \cdot \nabla v^{+} \geqslant\left|\nabla v^{+}\right|^{2}$ which is obvious (and equality holds). A similar remark holds for (5.2), whose classical counterpart is that if $-\Delta v=f$ in $\Omega$ and $v \leqslant 0$ on $\partial \Omega$ then one may test the equation against $w:=v^{+}$and obtain

$$
\int_{\Omega}|\nabla w|^{2} d x=\int_{\Omega} \nabla v \cdot \nabla w d x=\int_{\Omega}(-\Delta v) w d x=\int_{\Omega} f w d x .
$$

Proof of Lemma 10: To check the claim in (5.1), since the role of $x$ and $y$ is symmetric, we can always suppose $v(x) \geqslant v(y)$. Also (5.1) is clearly an identity when $x, y \in\{v \geqslant 0\}$ and when $x, y \in\{v<0\}$. So, it only remains to check (5.1) when $x \in\{v \geqslant 0\}$ and $y \in\{v<0\}$. In this case

$$
v^{+}(x)-v^{+}(y)=v(x)<v(x)+|v(y)|=v(x)-v(y),
$$

and so, if we multiply by $v^{+}(x)-v^{+}(y) \geqslant 0$ both sides, we obtain (5.1).

Proof of Lemma 11: By construction $v^{+}=0$ outside $\Omega$, so that $v^{+} \in$ $\mathbb{H}_{0}^{s}(\Omega)$, hence we can use it as a test function. Using this and Lemma 10 
we obtain

$$
\begin{aligned}
\int_{\mathbb{R}^{2 n}} \frac{|w(x)-w(y)|^{2}}{|x-y|^{n+2 s}} d x d y & =\int_{\mathbb{R}^{2 n}} \frac{\left|v^{+}(x)-v^{+}(y)\right|^{2}}{|x-y|^{n+2 s}} d x d y \\
& \leqslant \int_{\mathbb{R}^{2 n}} \frac{(v(x)-v(y))\left(v^{+}(x)-v^{+}(y)\right)}{|x-y|^{n+2 s}} d x d y \\
& =\int_{\Omega} f(x) v^{+}(x) d x,
\end{aligned}
$$

which concludes the proof of Lemma 11.

5.1. Proof of Proposition 9. We use the same arguments used in [14, Proposition 4] in order to prove a $L^{\infty}$-regularity result for the eigenfunctions of the operator $(-\Delta)^{s}$. The argument is a fractional version of the classical De Giorgi-Stampacchia iteration method.

By extending $f$ in a bounded way outside $\Omega$, we may suppose that $f \in L^{\infty}\left(\mathbb{R}^{n}\right)$. Moreover, we may assume that $u$ does not vanish identically (otherwise there is nothing to prove). Let $\delta>0$, to be taken appropriately small in what follows (the choice of $\delta$ will be done on (5.12) below). Up to multiplying $u$ by a small constant, we may and do assume that

$$
\|g\|_{L^{\infty}\left(\mathbb{R}^{n}\right)} \leqslant \frac{1}{2}
$$

(here we use the fact that $u=g$ a.e. in $\mathbb{R}^{n} \backslash \Omega$ ) and

$$
\|u\|_{L^{2}(\Omega)} \leqslant \sqrt{\delta}
$$

Now, for any $k \in \mathbb{N}$, we let $C_{k}:=1-2^{-k}, v_{k}:=u-C_{k}, w_{k}:=v_{k}^{+}$ and $U_{k}:=\left\|w_{k}\right\|_{L^{2}(\Omega)}^{2}$.

We remark that

$$
0 \leqslant w_{k} \leqslant|u|+\left|C_{k}\right| \leqslant|u|+1 \in L^{2}(\Omega),
$$

being $\Omega$ bounded, and

$$
w_{k} \rightarrow(u-1)^{+} \quad \text { a.e. in } \mathbb{R}^{n}
$$

as $k \rightarrow+\infty$. Therefore, by the Dominated Convergence Theorem applied in $L^{2}(\Omega)$, we get

$$
U_{k}:=\left\|w_{k}\right\|_{L^{2}(\Omega)}^{2} \rightarrow\left\|(u-1)^{+}\right\|_{L^{2}(\Omega)}^{2}
$$

as $k \rightarrow+\infty$. 
Moreover, for any $k \in \mathbb{N}$ we have that $C_{k+1}>C_{k}$ and so $v_{k+1}<v_{k}$ a.e. in $\mathbb{R}^{n}$ from which we deduce that

$$
w_{k+1} \leqslant w_{k} \quad \text { a.e. in } \mathbb{R}^{n} .
$$

Furthermore, by (5.3), in $\mathbb{R}^{n} \backslash \Omega$ we have that

$$
v_{k+1}=g-1+2^{-k-1} \leqslant-\frac{1}{2}+2^{-k-1} \leqslant 0,
$$

and $(-\Delta)^{s} v_{k+1}=(-\Delta)^{s} u=f$ in $\Omega$ in the weak sense. Therefore, we can apply Lemma 11 with $v:=v_{k+1}$ and $w:=v_{k+1}^{+}=w_{k+1}$, which gives that

$$
\begin{aligned}
\int_{\Omega \times \Omega} \frac{\left|w_{k+1}(x)-w_{k+1}(y)\right|^{2}}{|x-y|^{n+2 s}} d x d y & \leqslant \int_{\mathbb{R}^{2 n}} \frac{\left|w_{k+1}(x)-w_{k+1}(y)\right|^{2}}{|x-y|^{n+2 s}} d x d y \\
& \leqslant \int_{\Omega} f(x) w_{k+1}(x) d x .
\end{aligned}
$$

As a consequence of this,

$$
\begin{aligned}
& \int_{\Omega \times \Omega} \frac{\left|w_{k+1}(x)-w_{k+1}(y)\right|^{2}}{|x-y|^{n+2 s}} d x d y \\
& \leqslant \int_{\Omega \cap\left\{w_{k+1}>0\right\}}|f(x)| w_{k+1}(x) d x \\
& \leqslant\|f\|_{L^{\infty}\left(\mathbb{R}^{n}\right)} \int_{\Omega \cap\left\{w_{k+1}>0\right\}} w_{k+1}(x) d x \\
& \leqslant\|f\|_{L^{\infty}\left(\mathbb{R}^{n}\right)} \int_{\Omega \cap\left\{w_{k+1}>0\right\}} w_{k}(x) d x \\
& \leqslant\|f\|_{L^{\infty}\left(\mathbb{R}^{n}\right)}\left|\Omega \cap\left\{w_{k+1}>0\right\}\right|^{1 / 2}\left\|w_{k}\right\|_{L^{2}(\Omega)} \\
& \leqslant\|f\|_{L^{\infty}\left(\mathbb{R}^{n}\right)}\left|\Omega \cap\left\{w_{k+1}>0\right\}\right|^{1 / 2} U_{k}^{1 / 2},
\end{aligned}
$$

thanks to (5.6), the regularity of $f$, Hölder inequality and the definition of $U_{k}$.

Now we claim that

$$
\left\{w_{k+1}>0\right\} \subseteq\left\{w_{k}>2^{-(k+1)}\right\} .
$$


Weak and Viscosity Solutions of the Fractional Laplace Equation 151

To establish this, we observe that if $x \in\left\{w_{k+1}>0\right\}$ then

$$
0<w_{k+1}(x)=v_{k+1}^{+}(x)=\max \left\{u(x)-C_{k+1}, 0\right\} .
$$

Hence $u(x)-C_{k+1}>0$. Accordingly

$$
v_{k}(x)=u(x)-C_{k}>C_{k+1}-C_{k}=2^{-(k+1)},
$$

hence $w_{k}(x):=v_{k}^{+}(x)=v_{k}(x)>2^{-(k+1)}$, which proves $(5.8)$.

As a consequence of (5.8), we obtain

$$
\begin{aligned}
U_{k} & =\left\|w_{k}\right\|_{L^{2}(\Omega)}^{2} \\
& \geqslant \int_{\Omega \cap\left\{w_{k}>2^{-(k+1)}\right\}} w_{k}^{2}(x) d x \\
& \geqslant 2^{-2(k+1)}\left|\Omega \cap\left\{w_{k} \geqslant 2^{-(k+1)}\right\}\right| \\
& \geqslant 2^{-2(k+1)}\left|\Omega \cap\left\{w_{k+1}>0\right\}\right|,
\end{aligned}
$$

so that

$$
\left|\Omega \cap\left\{w_{k+1}>0\right\}\right| \leqslant 2^{2(k+1)} U_{k} .
$$

Then by (5.7) and (5.9) we get that

$$
\int_{\Omega \times \Omega} \frac{\left|w_{k+1}(x)-w_{k+1}(y)\right|^{2}}{|x-y|^{n+2 s}} d x d y \leqslant 2^{k+1}\|f\|_{L^{\infty}\left(\mathbb{R}^{n}\right)} U_{k}
$$

Now we use the Hölder inequality (with exponents $2^{*} / 2$ and $n /(2 s)$ ) and the fractional Sobolev inequality (see, e.g., Theorem 6.7 in $[6]$ ) to get that

$$
\begin{aligned}
U_{k+1} & \leqslant\left(\int_{\Omega}\left|w_{k+1}(x)\right|^{2^{*}} d x\right)^{2 / 2^{*}}\left|\Omega \cap\left\{w_{k+1}>0\right\}\right|^{2 s / n} \\
& \leqslant \tilde{c}\left|\Omega \cap\left\{w_{k+1}>0\right\}\right|^{2 s / n} \int_{\Omega \times \Omega} \frac{\left|w_{k+1}(x)-w_{k+1}(y)\right|^{2}}{|x-y|^{n+2 s}} d x d y
\end{aligned}
$$


for some positive constant $\tilde{c}$ depending only on $n$ and $s$. Consequently, by (5.9) and (5.10), we see that

$$
\begin{aligned}
U_{k+1} & \leqslant \tilde{c} 2^{k+1}\|f\|_{L^{\infty}\left(\mathbb{R}^{n}\right)} U_{k}\left(2^{2(k+1)} U_{k}\right)^{2 s / n} \\
& =\tilde{c}\|f\|_{L^{\infty}\left(\mathbb{R}^{n}\right)} 2^{1+4 s / n}\left(2^{1+4 s / n}\right)^{k} U_{k}^{1+2 s / n} \\
& \leqslant\left(1+\tilde{c}\|f\|_{L^{\infty}\left(\mathbb{R}^{n}\right)} 2^{1+4 s / n}\right)\left(2^{1+4 s / n}\right)^{k} U_{k}^{1+2 s / n} \\
& \leqslant\left[\left(1+\tilde{c}\|f\|_{L^{\infty}\left(\mathbb{R}^{n}\right)} 2^{1+4 s / n}\right) 2^{1+4 s / n}\right]^{k} U_{k}^{1+2 s / n} \\
& =C^{k} U_{k}^{\beta},
\end{aligned}
$$

where $\beta:=1+(2 s / n)>1$ and $C>1$ only depends on $n, s$ and $f$.

Now, we are ready to perform our choice of $\delta$ : namely we assume that $\delta>0$ is so small that

$$
\delta^{\beta-1}<\frac{1}{C^{1 /(\beta-1)}} .
$$

We also fix

$$
\eta \in\left(\delta^{\beta-1}, \frac{1}{C^{1 /(\beta-1)}}\right) .
$$

Notice that, since $C>1$ and $\beta>1$

$$
\eta \in(0,1) .
$$

Moreover

$$
\delta^{\beta-1} \leqslant \eta \quad \text { and } \quad C \eta^{\beta-1} \leqslant 1 .
$$

We claim that for any $k \in \mathbb{N}$

$$
U_{k} \leqslant \delta \eta^{k} .
$$

The proof is by induction. First of all, by (5.4)

$$
U_{0}:=\left\|w_{0}\right\|_{L^{2}(\Omega)}^{2}=\left\|u^{+}\right\|_{L^{2}(\Omega)}^{2} \leqslant\|u\|_{L^{2}(\Omega)}^{2} \leqslant \delta,
$$

which is (5.15) when $k=0$. Now, let us suppose that (5.15) holds true for $k$ and let us prove it for $k+1$. For this, we use (5.11) and (5.14):

$$
U_{k+1} \leqslant C^{k} U_{k}^{\beta} \leqslant C^{k}\left(\delta \eta^{k}\right)^{\beta}=\delta\left(C \eta^{\beta-1}\right)^{k} \delta^{\beta-1} \eta^{k} \leqslant \delta \eta^{k+1} .
$$

This proves (5.15). 
Then, by (5.13) and (5.15), we conclude that

$$
\lim _{k \rightarrow+\infty} U_{k}=0
$$

Hence, by $(5.5),(u-1)^{+}=0$ a.e. in $\Omega$, that is $u \leqslant 1$ a.e. in $\Omega$. By replacing $u$ with $-u$, we obtain $\|u\|_{L^{\infty}(\Omega)} \leqslant 1$. This ends the proof of Proposition 9 .

\section{References}

[1] G. Barles, E. Chasseigne, And C. Imbert, On the Dirichlet problem for second-order elliptic integro-differential equations, Indiana Univ. Math. J. 57(1) (2008), 213-246. DOI: 10.1512/iumj. 2008.57.3315.

[2] B. Barrios Barrera, A. Figalli, and E. Valdinoci, Bootstrap regularity for integro-differential operators and its application to nonlocal minimal surfaces, Ann. Sc. Norm. Super. Pisa Cl. Sci. (5) (to appear). DOI: 10.2422/2036-2145.201202_007.

[3] L. Caffarelli and L. Silvestre, An extension problem related to the fractional Laplacian, Comm. Partial Differential Equations 32(7-9) (2007), 1245-1260. DOI: 10.1080/03605300600987306.

[4] L. Caffarelli and L. Silvestre, Regularity theory for fully nonlinear integro-differential equations, Comm. Pure Appl. Math. 62(5) (2009), 597-638. DOI: 10.1002/cpa.20274.

[5] L. Caffarelli and L. Silvestre, Regularity results for nonlocal equations by approximation, Arch. Ration. Mech. Anal. 200(1) (2011), 59-88. DOI : 10.1007/s00205-010-0336-4.

[6] E. Di Nezza, G. Palatucci, and E. Valdinoci, Hitchhiker's guide to the fractional Sobolev spaces, Bull. Sci. Math. 136(5) (2012), 521-573. DOI: 10.1016/j.bulsci.2011.12.004.

[7] H. Dong AND D. KIm, On $L_{p}$-estimates for a class of non-local elliptic equations, J. Funct. Anal. 262(3) (2012), 1166-1199. DOI: $10.1016 / \mathrm{j} \cdot \mathrm{jfa} .2011 .11 .002$.

[8] D. Gilbarg And N. S. TRudinger, "Elliptic partial differential equations of second order", Reprint of the 1998 edition, Classics in Mathematics, Springer-Verlag, Berlin, 2001.

[9] N. S. LANDKOF, "Foundations of modern potential theory", Translated from the Russian by A. P. Doohovskoy, Die Grundlehren der mathematischen Wissenschaften 180, Springer-Verlag, New YorkHeidelberg, 1972. 
[10] X. Ros-Oton and J. Serra, Fractional Laplacian: Pohozaev identity and nonexistence results, C. R. Math. Acad. Sci. Paris 350(9-10) (2012), 505-508. DOI: 10.1016/j.crma.2012.05.011.

[11] X. Ros-Oton and J. SerRa, The Dirichelt problem for the fractional Laplacian: regularity up to the boundary, personal communication.

[12] R. Servadei and E. Valdinoci, Mountain pass solutions for non-local elliptic operators, J. Math. Anal. Appl. 389(2) (2012), 887-898. DOI: $10.1016 / j . j m a a .2011 .12 .032$.

[13] R. Servadei and E. Valdinoci, Variational methods for nonlocal operators of elliptic type, Discrete Contin. Dyn. Syst. 33(5) (2013), 2105-2137. DOI: 10.3934/dcds.2013.33.2105.

[14] R. Servadei and E. Valdinoci, A Brezis-Nirenberg result for non-local critical equations in low dimension, Commun. Pure Appl. Anal. 12(6) (2013), 2445-2464. DOI: 10.3934/cpaa.2013.12.2445.

[15] L. Silvestre, Regularity of the obstacle problem for a fractional power of the Laplace operator, $\mathrm{PhD}$ Thesis, University of Texas at Austin (2005). http://math.uchicago.edu/ luis/ preprints/luisdissreadable.pdf.

Raffaella Servadei:

Dipartimento di Matematica e Informatica

Università della Calabria

Ponte Pietro Bucci 31 B

87036 Arcavacata di Rende (Cosenza)

Italy

E-mail address: servadei@mat.unical.it

Enrico Valdinoci:

Dipartimento di Matematica

Università di Milano

Via Cesare Saldini 50

20133 Milano

Italy

and

Weierstraß-Institut für Angewandte Analysis und Stochastik

Mohrenstraße 39

10117 Berlin

Germany

E-mail address: enrico.valdinoci@unimi.it

Primera versió rebuda el 5 de setembre de 2012, darrera versió rebuda el 3 de desembre de 2012. 